\title{
Isothermal Compressibility of Monatomic Liquids and Binary Mixtures
}

\author{
By K. Takesawa*, S. Takeda** and S. Tamaki***
}

\begin{abstract}
The isothermal compressibility for the monatomic and binary liquids is discussed in terms of pair potential or direct correlation function. In Lennard-Jones fluids, the pair potential is divided into two parts; one is the short-range repulsive part which is replaced with the hard sphere one and the other is the long range attractive part for which an idea of homogeneous mean field approach is taken into account. The calculation has been applied to $\mathrm{Ne}, \mathrm{Ar}, \mathrm{Kr}$ and $\mathrm{Xe}$, and binary mixtures of $\mathrm{Ar}-\mathrm{Kr}$ and $\mathrm{Kr}-\mathrm{Xe} \mathrm{In}$ liquid metals, the isothermal compressibility is closely related to the atomic core size and the screening effect of the conduction electrons. The actual calculation has been carried out for liquid $\mathrm{Na}$ and $\mathrm{K}$, and their binary mixture.
\end{abstract}

(Received August 27, 1986)

Keywords: compressibility, liquids, binary mixtures, pair potential, density fluctuatıon

\section{Introduction}

It is well known that there is, in the liquid state, an equivalence among the isothermal compressibility, $\chi_{\mathrm{T}}$, the structure factor in the long wavelength limit $a(q=0)$ and density fluctuation in the long wavelength limit. A crude model can be obtained for certain monatomic liquid by Percus-Yevick hard sphere approximation $^{(1)}$, and the compressibility under the same condition can be expressed by the following form,

$$
\chi_{\mathrm{T}}^{\mathrm{hs}}=a_{\mathrm{hs}}(q=0) / n k_{\mathrm{B}} T
$$

with

$$
a_{\mathrm{hs}}(q=0)=(1-\eta)^{4}(1+2 \eta)^{-2},
$$

where $\eta$ is the packing fraction of the hard sphere and $n$ is the number density. Evans and Schirmacher ${ }^{(2)}$ have derived a theory of compressibility of adding the attractive part of the effective pair potential to $a_{\mathrm{hs}}(q=0)$ of the hard sphere system and obtained the following expression for the structure factor in the long wavelength limit, $a_{\mathrm{RES}}(q=0)$,

\footnotetext{
* Nigata Minamı High School, Niıgata 950, Japan

** College of Bio-Medical Technology, Ningata Unıversity, Nugata 951, Japan.

*** Department of Physics, Faculty of Science, Nugata University, Ni1gata 950-21, Japan.
}

$$
\begin{aligned}
a_{\mathrm{RES}}(q=0)= & a_{\mathrm{hs}}(q=0)\left[1+\beta n a_{\mathrm{hs}}(q=0)\right. \\
& \left.\times \int \phi_{1}(r) g_{\mathrm{hs}}(r) \mathrm{d} r\right]^{-1},
\end{aligned}
$$

where $\beta=1 / k_{\mathrm{B}} T, \phi_{1}(r)$ the attractive potential under the WCA prescription ${ }^{(3)}$ and $g_{\mathrm{hs}}(r)$ the pair distribution function of hard spheres. Their results fairly agree with the experimental data, but there is still some disagreement between the calculations and experiments.

The compressibility for monatomic liquids can be estimated from the pressure equation in which the pair distribution function or the structure factor is used ${ }^{(4)-(6)}$.

The purpose of this paper is to present a method of calculating the isothermal compressibility, $\chi_{\mathrm{T}}$, of Lennard-Jones fluids, liquid metals and their mixtures using the pair potential representation. In Lennard-Jones liquids, the pair potential in the systems are divided into two parts; the short-range repulsive part which is replaced with the hard sphere one and the long-range attractive part which is given by the homogeneous mean field. The results of liquid $\mathrm{Ar}$ and $\mathrm{Kr}$ are in good agreement with the observed data. The application to the mixture of Lennard-Jones fluids as $\mathrm{Ar}-\mathrm{Kr}$ and $\mathrm{Kr}-\mathrm{Xe}$ indicates an interesting result as shown in the following section.

The application is extended to the simple 
liquid metals such as $\mathrm{Na}$ and $\mathrm{K}$, and these alloys. It has been proved that the isothermal compressibility in liquid metals is closely related to the atomic core size and the screening effect of the conduction electrons.

\section{Compressibility for Pure Liquids}

The pair distribution function $g(r)$ is connected with the total correlation function $h(r)$ as

$$
h(r) \equiv g(r)-1 .
$$

The total correlation function is conveniently regarded as being composed of a direct $c(r)$ and an indirect term which describes the correlation of the pair considered through the action of a third particle. Thus $c(r)$ is defined by

$$
h\left(r_{12}\right)=c\left(r_{12}\right)+n \int c\left(r_{13}\right) h\left(r_{23}\right) \mathrm{d} r_{3},
$$

which is known as the Ornstein-Zernike relation $^{(7)}$. This equation defines only $c(r)$ whose range of interaction is expected to be smaller than that of $h(r)$.

For the total correlation function $h(r)$, the following relation is known as the compressibility equation $^{(9)}$;

$$
n k_{\mathrm{B}} T \chi_{\mathrm{T}}=1+n \int h(r) \mathrm{d} r
$$

On the other hand, the total correlation function $h(r)$ can be expressed as

$$
a(q)=1+n \int h(r) \exp (i q \cdot r) \mathrm{d} r .
$$

Comparing eq. (6) with eq. (7), we have

$$
a(q=0)=n k_{\mathrm{B}} T \chi_{\mathrm{T}} .
$$

Taking the Fourier transform of both sides of (5) and using the convolution theorem, we find

$$
a(q)-1=n c(q)+n c(q)[a(q)-1]
$$

or

$$
a(q)=1 /[1-n c(q)] \text {. }
$$

From eqs. (8) and (10), we have

$$
\chi_{\mathrm{T}}=\frac{1}{n k_{\mathrm{B}} T[1-n c(q=0)]} .
$$

\section{Extension to the Binary Liquid Mixture}

Ornstein-Zernike equation can be readily extended to the binary liquid system composed of number densities $n_{1}$ of the component 1 and $n_{2}$ of 2 (hereafter referred to as $n_{i}$ ) as ${ }^{(8)}$

$$
\begin{aligned}
h_{i j}(r)= & c_{i j}(r)+\sum_{l=1}^{2} n_{l} \int h_{i l}\left(r^{\prime}\right) \\
& \times c_{l j}\left(\left|r^{\prime}-r\right|\right) \mathrm{d} r^{\prime}(i, j=1,2)
\end{aligned}
$$

and

$$
h_{i j}(r) \equiv g_{i j}(r)-1,
$$

defining the Fourier transforms of $c_{i j}(r)$ and $h_{i j}(r)$ as follows;

$$
c_{i j}(q)=\int_{0}^{\infty} c_{i j}(r) \frac{\sin (q r)}{q r} 4 \pi r^{2} \mathrm{~d} r
$$

and

$$
a_{i j}(q)-1=n \int_{0}^{\infty} h_{i j}(r) \frac{\sin (q r)}{q r} 4 \pi r^{2} \mathrm{~d} r
$$

where $a_{i j}(q)$ is so-called Faber-Ziman type partial structure factors. Then we have

$$
\begin{aligned}
& a_{11}(q)= {\left[1+n x_{2}\left\{c_{11}(q)-c_{22}(q)\right\}\right.} \\
&\left.-n^{2} x_{2}^{2}\left\{c_{11}(q) c_{22}(q)-c_{12}^{2}(q)\right\}\right] / D, \\
& a_{22}(q)= {\left[1+n x_{1}\left\{c_{22}(q)-c_{11}(q)\right\}\right.} \\
&\left.-n^{2} x_{1}^{2}\left\{c_{11}(q) c_{22}(q)-c_{12}^{2}(q)\right\}\right] / D, \\
& a_{12}(q)= a_{21}(q)=1+n c_{12}(q) / D \\
& \text { and }
\end{aligned}
$$

$$
\begin{aligned}
D= & {\left[1-n x_{1} c_{11}(q)\right]\left[1-n x_{2} c_{22}(q)\right] } \\
& -n^{2} x_{1} x_{2} c_{12}^{2}(q)
\end{aligned}
$$

with

$$
x_{i}=n_{i} /\left(n_{1}+n_{2}\right) \quad(i=1,2) .
$$

On the other hand, the partial structure factors $S_{i j}(q)$ proposed by Ashcroft and Langreth $^{(9)}$ (hereafter referred to as A-L type) are

$$
S_{i j}(q)=\delta_{i j}+\left(x_{i} x_{j}\right)^{1 / 2}\left[a_{i j}(q)-1\right] .
$$

Putting the eq. (15) into (17), we find the following relations; 


$$
\begin{aligned}
& S_{11}(q)=\left[1-n x_{2} c_{22}(q)\right] / D, \\
& S_{22}(q)=\left[1-n x_{2} c_{11}(q)\right] / D
\end{aligned}
$$

and

$$
S_{12}(q)=n \sqrt{x_{1} x_{2}} c_{12}(q) / D .
$$

Kirkwood and Buff ${ }^{(10)}$ have obtained the isothermal compressibility, $\chi_{\mathrm{T}}$, of the liquid system $A_{x_{1}} B_{x_{2}}$ as follows;

$$
\chi_{\mathrm{T}}=\frac{\beta}{n} \frac{S_{11} S_{22}-S_{12}^{2}}{x_{1} S_{22}+x_{2} S_{11}-2\left(x_{1} x_{2}\right)^{1 / 2} S_{12}},
$$

where $S_{\imath \jmath} \equiv S_{l j}(q=0)$. If one notices that the numerator of (19) equals to $D^{-1}$, the isothermal compressibility, $\chi_{\mathrm{T}}$, can be expressed in terms of the Fourier transform of the direct correlation function $c_{l j}(q)$ as follows;

$$
\chi_{\mathrm{T}}=\frac{\beta}{n} \frac{1}{\left[1-n\left(x_{1}^{2} c_{11}+2 x_{1} x_{2} c_{12}+x_{2}^{2} c_{22}\right)\right]},
$$

where $c_{l j} \equiv c_{l}(q=0)$. This expression is an extension of the isothermal compressibility to the binary liquid mixture.

According to the Percus-Yevick approximation, the direct correlation function $c_{l j}(r)$ is given by the following equation (see for example March and Tosi $^{(7)}$ );

$$
c_{l j}(r)=g_{l j}(r)\left[1-\exp \left(\beta \phi_{l J}(r)\right)\right] .
$$

At the large distance $r$, where $\phi_{l j}(r) / k_{\mathrm{B}} T$ tends to be much smaller than unity, eq. (21) is approximately given by

$$
c_{l j}(r)=-\beta g_{\imath \jmath}(r) \phi_{l j}(r)
$$

and

$$
c_{l j}(r) \sim-\beta \phi_{l j}(r)
$$

for large $r$.

\section{Calculation of Isothermal Compressibility}

\section{Lennard-Jones fluids}

Interatomic forces can be divided into two parts; one is a steep short-range repulsive force and the other is a smoothly varying long-range attractive one. Therefore, we divided the pairwise interatomic potential, $\phi(r)$, into a repulsive part, $\phi_{s r}(r)$, and an attractive one, $\phi_{l r}(r)$. Herewith suffixes $s r$ and $l r$ mean the short-range force and the long-range one, respectively.

For the application of eq. (21) to a LennardJones fluid, we approximate for the simplicity that the steep repulsive part is represented by hard core potential with the hard sphere diameter, $\sigma$, and that the attractive part is represented as Lennard-Jones potential with the depth of its potential at the minimum, $\varepsilon$. This pairwise potential can be written as,

$$
\phi(r)=\left\{\begin{array}{ll}
\phi_{s r}(r)=+\infty & r \leq \sigma \\
\phi_{l r}(r)=4 \varepsilon\left[(\sigma / r)^{12}-(\sigma / r)^{6}\right] & r>\sigma
\end{array} .\right.
$$

From eqs. (21) and (24), we have

$$
\begin{aligned}
c(q \rightarrow 0)= & \int_{0}^{\sigma} c(r) 4 \pi r^{2} d r+\int_{\sigma}^{\infty} g(r) \\
& \times\left[1-\exp \left(\beta \phi_{l r}(r)\right)\right] 4 \pi r^{2} \mathrm{~d} r .
\end{aligned}
$$

The first term of the right hand side of eq. (25) can be taken as long wavelength limit of the direct correlation function $c(q)$ for a hard sphere liquid, say $c^{\text {hs }}(q=0)$. Wertheim and Thiele obtained independently the exact $c^{\text {hs }}(q=0)$ as

$$
\begin{aligned}
c^{\mathrm{hs}}(q \rightarrow 0) & =\left[1-\frac{1}{a^{\mathrm{hs}}(0)}\right] / n \\
& =\left[1-\frac{1}{n k_{\mathrm{B}} T \chi \chi_{\mathrm{T}}^{\mathrm{hs}}}\right] / n .
\end{aligned}
$$

Because of a condition $\left|\beta \phi_{l r}(r)\right| / 2<1$ in the present case, the second term of the right hand side of eq. (25) is roughly transformed into

$$
c^{l r}(q \rightarrow 0) \simeq-\beta \int_{\sigma}^{\infty} \phi_{l r} g(r) 4 \pi r^{2} \mathrm{~d} r .
$$

The right hand side of eq. (27) can be rewritten as

$$
\begin{aligned}
& -4 \pi \beta \int_{\sigma}^{\infty} \phi_{l r}(r) r^{2} \mathrm{~d} r \\
& -4 \pi \beta \int_{\sigma}^{\infty} \phi_{l r}(r)[g(r)-1] r^{2} \mathrm{~d} r .
\end{aligned}
$$

The first term of (27)' corresponds to the homogeneous meanfield term in the long-range potential part and the second one is its deviation due to the density fluctuation. Applying 
Table 1 The used parameters, and the calculated and observed values of isothermal compressibilities.

\begin{tabular}{|c|c|c|c|c|c|c|c|c|c|c|c|}
\hline & \multirow{2}{*}{$\begin{array}{c}n \\
\left(\mathrm{~A}^{-3}\right)\end{array}$} & \multirow{2}{*}{$\begin{array}{c}\varepsilon \\
\left(10^{-2} \mathrm{eV}\right)\end{array}$} & \multirow{2}{*}{$\begin{array}{c}\sigma \\
(\mathrm{A})\end{array}$} & \multirow{2}{*}{$\begin{array}{c}T \\
(\mathrm{~K})\end{array}$} & \multicolumn{3}{|c|}{$\begin{array}{l}\text { Each term of denomina- } \\
\text { tor in }(29)\left(10^{8} \mathrm{~N} \cdot \mathrm{m}^{-2}\right)\end{array}$} & $\chi_{\mathrm{T}}^{\mathrm{hs}}$ & & & $\chi_{\mathrm{T}}^{\mathrm{E}-5 * *}$ \\
\hline & & & & & First & Second & Third & \multicolumn{4}{|c|}{$\left(10^{-9} \mathrm{~m}^{2} \cdot \mathrm{N}^{-1}\right)$} \\
\hline $\mathrm{Ne}$ & 0.0373 & 0.0306 & 2.75 & 25 & 3.40 & -1.58 & -0.56 & 2.94 & 7.94 & 4.04 & 4.28 \\
\hline $\mathrm{Ar}$ & 0.0210 & 1.03 & 3.40 & 85 & 8.24 & -3.20 & -0.74 & 1.21 & 2.35 & 2.03 & 1.79 \\
\hline $\mathrm{Kr}$ & 0.0175 & 1.41 & 3.63 & 117 & 9.96 & -3.68 & -0.15 & 1.01 & 1.66 & 1.75 & 1.24 \\
\hline $\mathrm{Xe}$ & 0.0161 & 1.94 & 4.07 & 161 & 48.79 & -6.79 & -0.14 & 0.205 & 0.239 & - & - \\
\hline
\end{tabular}

* present calculation by using (29)

** by Evans and Schirmacher ${ }^{(2)}$

eq. (24), we have the first term of eq. (27)' as

$$
4 \pi \beta \int_{\sigma}^{\infty} \phi_{l r}(r) r^{2} \mathrm{~d} r=-(32 / 9) \pi \varepsilon \beta \sigma^{3} .
$$

Substituting eqs. (26)-(28) for (11), we have

$$
\begin{aligned}
\chi_{\mathrm{T}} & =\frac{\beta}{n} \frac{1}{1-n c^{\mathrm{hs}}(q \rightarrow 0)-\frac{32}{9} n \pi \varepsilon \beta \sigma^{3}+4 n \pi \beta \int_{\sigma}^{\infty} \phi_{\mathrm{lr}}(r)[g(r)-1] r^{2} \mathrm{~d} r} \\
& =\frac{1}{\frac{1}{\chi_{\mathrm{T}}^{\mathrm{hs}}}-\frac{32}{9} n^{2} \pi \varepsilon \sigma^{3}+4 n^{2} \pi \beta \int_{\sigma}^{\infty} \phi_{l r}(r)[g(r)-1] r^{2} \mathrm{~d} r} .
\end{aligned}
$$

We use the parameters $\varepsilon$ and $\sigma$ of the LennardJones fluids listed in the work of Hansen and $\mathrm{McDonald}^{(11)}$, in which the numerical values are determined from the second virial coefficient measurements and the corresponding reduced critical constants, and also we use the observed distribution functions ${ }^{(12)-(15)}, g(r)$. A better agreement between the calculated and observed values of $\chi_{\mathrm{T}}$ 's can be seen in Table 1.

\section{Mixture of Lennard-Jones fluids}

In the case of binary system, the van der Waals' pair potential between component $i$ and $j$ atoms may be also written as follows;

$\phi_{i j}(r)=\left\{\begin{array}{ll}+\infty & r<\sigma_{i j} \\ 4 \varepsilon_{i j}\left[\left(\sigma_{i j} / r\right)^{12}-\left(\sigma_{i j} / r\right)^{6}\right] & r \geq \sigma_{i j}\end{array}\right.$,

assuming that the parameter $\varepsilon_{i j}$ and $\sigma_{i j}$ in this equation are approximated as follows ${ }^{(11)}$,

$$
\varepsilon_{i j} \simeq\left(\varepsilon_{i} \varepsilon_{j}\right)^{1 / 2}
$$

and

$$
\sigma_{i j} \simeq\left(\sigma_{i}+\sigma_{j}\right) / 2 .
$$

In the case of a liquid mixture, the isothermal compressibility of binary system $A_{x_{1}} B_{x_{2}}$ is written as follows, on the assumption that the terms corresponding to the last one of the denominator in eq. (29) are negligibly small compared with the terms of eq. (34);

$$
\chi_{\mathrm{T}}(\text { binary })=\frac{\beta}{n} \frac{1}{[A]}
$$

with

$$
\begin{aligned}
{[A]=} & 1-n\left[c_{11}^{\mathrm{hs}}(q \rightarrow 0)+c_{12}^{\mathrm{hs}}(q \rightarrow 0)+c_{22}^{\mathrm{hs}}(q \rightarrow 0)\right] \\
& +n \beta\left\{x_{1}^{2}\left[\phi_{l r}(1-1)\right]+2 x_{1} x_{2}\left[\phi_{l r}(1-2)\right]\right. \\
& \left.+x_{2}^{2}\left[\phi_{l r}(2-2)\right]\right\},
\end{aligned}
$$

where

$$
\begin{aligned}
{\left[c_{i j}^{\mathrm{hs}}(q \rightarrow 0)\right] } & =\left[1-a_{i j}^{\mathrm{hs}}(0)^{-1}\right] / n \\
& =\left[1-\left(n k_{\mathrm{B}} T \chi_{\mathrm{T}}^{\mathrm{hs}}\right)^{-1}\right] / n,
\end{aligned}
$$




$$
\begin{aligned}
{\left[\phi_{l r}(i-j)\right] } & =4 \pi \int_{\sigma_{l y}}^{\infty} \phi_{l r}(i-j) r^{2} \mathrm{~d} r \\
& =-(32 / 9) \pi \varepsilon_{l j} \sigma_{l j}^{3}
\end{aligned}
$$

and $x_{1}+x_{2}=1$.

The first bracket term on the right hand side of eq. (32) corresponds to that of hard sphere mixture and is written as $(n / \beta) \chi_{\mathrm{T}}^{\mathrm{hs}}$ (binary). According to Ashcroft and Langreth ${ }^{(9)}$, $\chi_{\mathrm{T}}^{\mathrm{hs}}$ (binary) is given by

$$
\chi_{\mathrm{T}}^{\mathrm{hs}}(\text { binary })=(\beta / n)(1-\eta)^{4}\left\{(1+2 \eta)^{2}-\Delta\right\}^{-1}
$$

with

$$
\begin{aligned}
\Delta= & \frac{3 x_{1} x_{2} \eta(1-\alpha)^{2}}{x_{1}+x_{2} \alpha^{3}}[(2+\eta)(1+\alpha)] \\
& +\frac{3 \eta \alpha\left(x_{2} \alpha^{2}+x_{1}\right)}{x_{1}+x_{2} \alpha^{3}}
\end{aligned}
$$

and

$$
\eta=\frac{x_{1} V_{1}}{V} \eta_{1}+\frac{x_{2} V_{2}}{V} \eta_{2}, \quad \alpha=\sigma_{1} / \sigma_{2} \quad\left(\sigma_{1}<\sigma_{2}\right),
$$

where $\sigma_{l}, V_{l}$ and $\eta_{l}$ are the hard sphere diameter, molar volume and packing fraction of atomic component $i$, respectively.

Putting eqs. (35) and (36) into (31), we have the following expression under the condition of random mixture.

$$
\chi_{\mathrm{T}}(\text { binary })=\frac{1}{\frac{1}{\chi_{\mathrm{T}}^{\text {hs }}(\text { binary })}-\frac{32}{9} n^{2} \pi\left[x_{1}^{2} \varepsilon_{11} \sigma_{1}^{3}+2 x_{1} x_{2} \varepsilon_{12} \sigma_{12}^{3}+x_{2}^{2} \varepsilon_{22} \sigma_{2}^{3}\right]} .
$$

Using the observed volume data for liquid Ar$\mathrm{Kr}$ mixture at $116 \mathrm{~K}^{(16)}$ and $\mathrm{Kr}-\mathrm{Xe}$ at $161 \mathrm{~K}^{(11)}$, in addition to the same parameter listed in Table 1, the estimated concentration curves of isothermal compressibilities of the van der Waals' mixture are shown in Figs. 1 and 2.

McAlister et al. ${ }^{(17)}$ reported that there is a preference of correlation between like atom pairs to that between unlike ones in these mix-

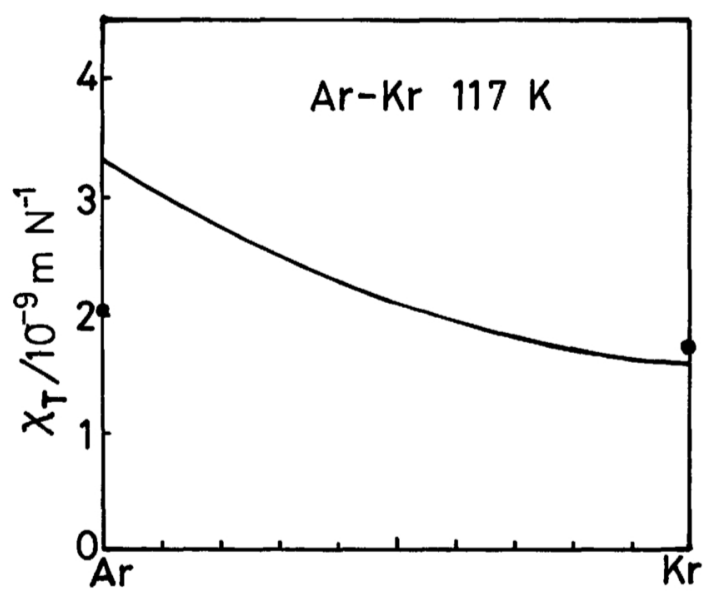

Fig. 1. Concentration variation of the isothermal compressibility of the binary liquid mixture $\mathrm{Ar}-\mathrm{Kr}$ at $117 \mathrm{~K}$ ; experimental values of liquid $\mathrm{Ar}$ at $85 \mathrm{~K}$ and liquid $\mathrm{Kr}$ at $117 \mathrm{~K}$. tures. So, the calculated concentration curve of isothermal compressibility from a linearly interpolated one may be slightly reduced by this trend. However, since any experimental data of the isothermal compressibilities of the mix-

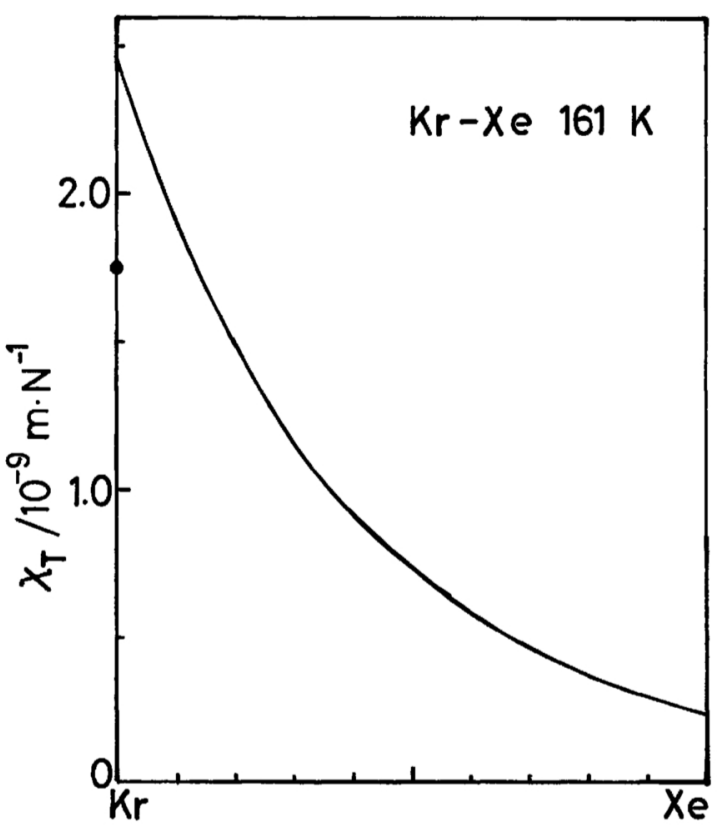

Fig. 2 Concentration variation of the isothermal compressibility of the binary liquid mixture $\mathrm{Kr}-\mathrm{Xe}$ at $161 \mathrm{~K}$ ; experimental value of hquid $\mathrm{Kr}$ at $117 \mathrm{~K}$. 
ture of Lennard-Jones fluids have not been obtained yet as far as the authors are concerned, the quantitative comparison between the theory and experimental one can not be made at the present time.

\section{Liquid Na-K Alloys}

If the electron-ion interaction is represented by a local pseudopotential, $V_{\mathrm{ps}}(r)$, the effective pairwise potential between component $i$ and $j$ atoms ${ }^{(18)}$ can be written as

$$
\phi_{i j}(q)=\frac{z_{i} z_{j}}{q^{2}} e^{2}+V_{\mathrm{ps}}^{i}(q) V_{\mathrm{ps}}^{j}(q) \chi^{e}(q),
$$

where $\chi^{\mathrm{e}}(q)$ is the density response function of the interacting homogeneous electron gas. For the simplicity in our calculation, we have employed the Hartree dielectric function and Ashcroft empty core model for the pseudopotential, respectively, as

$$
\chi^{e}(q) \equiv\left[1 / \varepsilon_{H}(q)-1\right]=\frac{-K_{\mathrm{TF}}^{2} /\left(2 q^{2}\right) \cdot \Pi(q)}{1+K_{\mathrm{TF}}^{2} /\left(2 q^{2}\right) \cdot \Pi(q)}
$$

with

$$
\Pi(q)=1+\frac{1-x^{2}}{2 x} \ln \left|\frac{1+x}{1-x}\right|, \quad\left(x=q / 2 k_{F}\right)
$$

and

$$
V_{\mathrm{ps}}^{i}(q)=-\frac{4 \pi z_{j} e^{2}}{q^{2}} \cos q R_{c}^{i},
$$

where $K_{\mathrm{TF}}$ is the Thomas-Fermi screening parameter defined as

$$
K_{\mathrm{TF}}=\left(\frac{6 \pi n e^{2}}{E_{\mathrm{F}}}\right)^{1 / 2}, \quad E_{\mathrm{F}}=\frac{\hbar^{2} k_{F}^{2}}{2 m}=\frac{\hbar^{2}\left(3 \pi n_{e}\right)^{2 / 3}}{2 m}
$$

and $n_{\mathrm{e}}, m$ and $R_{\mathrm{c}}^{i}$ are the number density, mass of electron and the empty core radius of ion $i$, respectively. Using these parameters, the direct correlation function in the long wavelength limit is written as

$$
c_{i j}(q \rightarrow 0)=4 \pi \int_{0}^{\infty} g_{i j}(r)\left[1-\exp \left(\beta \phi_{i j}(r)\right] r^{2} \mathrm{~d} r\right.
$$

$$
=4 \pi \int_{0}^{r_{0}} g_{i j}(r)\left[1-\exp \left(\beta \phi_{i j}(r)\right] r^{2} \mathrm{~d} r+4 \pi \int_{r_{0}}^{\infty} g_{i j}(r)\left[1-\exp \left(\beta \phi_{i j}(r)\right] r^{2} \mathrm{~d} r,\right.\right.
$$

where $r_{0} \sim e^{2} / k_{\mathrm{B}} T\left(\ll R_{\mathrm{c}}^{i}\right)$. Since the radial distribution function $g_{i j}(r)$ 's are very much small in the range of $0<r<r_{0}$, we can approximate the eq. (44) to

$$
\begin{aligned}
(1 / \beta) c_{i j}(q \rightarrow 0) & \simeq-4 \pi \int_{0}^{\infty} \phi_{i j}(r) q_{i j}(r) r^{2} \mathrm{~d} r \\
& =-\phi_{i j}(q \rightarrow 0)-4 \pi \int_{0}^{\infty} \phi_{i j}(r)\left[g_{i j}(r)-1\right] r^{2} \mathrm{~d} r .
\end{aligned}
$$

In the case of the empty-core potential which can be used for the construction of the effective pair potentials in liquid alloys, the first term on the right hand side of eq. (46) is given by the following form from eq. (38) to (41);

$$
\phi_{i j}(q \rightarrow 0)=2 \pi e^{2}\left[\left(R_{\mathrm{c}}^{i}\right)^{2}+\left(R_{\mathrm{c}}^{j}\right)^{2}+2\left(1 / K_{\mathrm{TF}}\right)^{2}\right] .
$$

Equation (47) has an instructive form, because $R_{\mathrm{c}}^{i}$ and $R_{\mathrm{c}}^{j}$ are related to their atomic core sizes and the effect of conduction electron which is taken into the Thomas-Fermi screening parameter. For the convenience of discussion, we will rewrite eq. (46) as follows;

$$
-(1 / \beta) c_{i j}(q \rightarrow 0)=2 \pi e^{2}\left[\left(R_{c}^{i}\right)^{2}+\left(R_{c}^{j}\right)^{2}+2\left(1 / K_{T F}\right)^{2}\right]+4 \pi \int_{0}^{\infty} \phi_{i j}(r)\left[q_{i j}(r)-1\right] r^{2} \mathrm{~d} r .
$$


Table 2 The used parameters, and the calculated and observed values isothermal compressibilities.

\begin{tabular}{|c|c|c|c|c|c|c|c|c|c|}
\hline & $\begin{array}{c}n \\
\left(\mathrm{~A}^{-3}\right)\end{array}$ & $\begin{array}{l}R_{\mathrm{c}} \\
\text { (A) }\end{array}$ & $\begin{array}{c}T \\
(\mathrm{~K})\end{array}$ & $4 \pi e^{2} R_{\mathrm{c}}^{2}$ & $\begin{array}{l}4 \pi e^{2} / K_{\mathrm{TF}}^{2} \\
0^{-37} \mathrm{~m}^{4} \mathrm{~N}\end{array}$ & $\begin{array}{c}\text { Integral } \\
\text { term in } \\
\quad(48)\end{array}$ & $\chi_{\mathrm{T}}^{\text {cal }^{*}}$ & $\begin{array}{r}\chi_{\mathrm{T}}^{\mathrm{exp}} \\
-{ }^{-11} \mathrm{~m}^{2}\end{array}$ & $\chi_{\mathrm{T}}^{\mathrm{E}-\mathrm{S}^{* *}}$ \\
\hline $\mathrm{Na}$ & 0.0243 & 0.900 & 371 & 2293 & 1.343 & -2.798 & 196 & 18.5 & 193 \\
\hline $\mathbf{K}$ & 00127 & 1.191 & 337 & 4100 & 1671 & -4.256 & 40.2 & 38.2 & 45.7 \\
\hline
\end{tabular}

If we use $R_{\mathrm{c}}$ 's derived by Umar et al. ${ }^{(19)}$ for $\mathrm{Na}$ and $\mathrm{K}$ and the $g_{\imath j}(r)$ 's observed by Waseda $^{(20)}$, then the isothermal compressibilities of liquid $\mathrm{Na}-\mathrm{K}$ alloys can be estimated by using eq. (20) and (48). The results are shown in Table 2 and Fig. 3 together with the experimental data ${ }^{(21)}$.

It is noteworthy to mention that the estimated values of the terms of $\left(R_{\mathrm{c}}^{l}\right)^{2}$ and $\left(1 / K_{\mathrm{TF}}\right)^{2}$

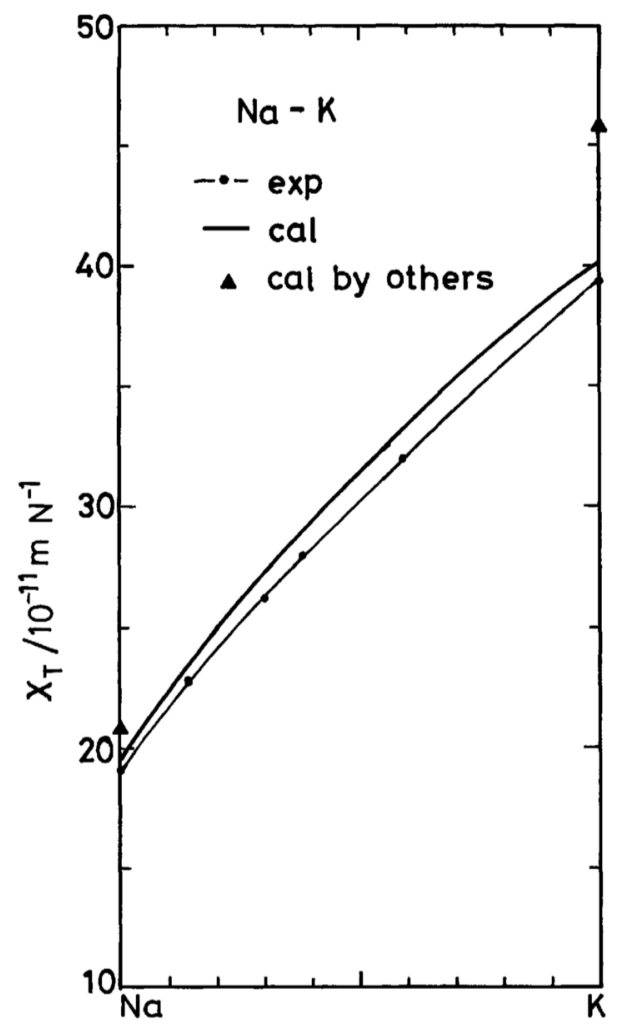

Fig. 3. Concentration variation of the isothermal compressibility of the liquid $\mathrm{Na}-\mathrm{K}$ alloys at $383 \mathrm{~K}\left(110^{\circ} \mathrm{C}\right)$, - - ; experımental values by Abowitz and Gordon ${ }^{(21)}$, solid curve; calculated by present authors, and $\boldsymbol{\Delta}$; calculated values by Evans and Schirmacher ${ }^{(2)}$. have the same order of magnitude. The agreement between the calculation and the experiments are qualitatively and quantitatively satisfactory.

\section{Discussion}

We adopted eqs. (24)-(29) for the LennardJones fluids and eqs. (43) to (48) for liquid metals as an approximation. This method is based on the fact that $g_{v j}(r) \phi_{l y}(r)$ is nearly equal to $-(1 / \beta) c_{l j}(r)$ above the melting point.

Using eqs. (1) and (28), eq. (29) is rewritten as follows;

$$
\chi_{\mathrm{T}}=\chi_{\mathrm{T}}^{\mathrm{hs}} \frac{1}{1+\chi_{\mathrm{T}}^{\mathrm{hs}} 4 n^{2} \pi \int_{\sigma}^{\infty} \phi_{l r}(r) g(r) r^{2} \mathrm{~d} r} .
$$

If we take $g(r)=g_{\mathrm{hs}}(r)$, then eq. (49) turns to a similar form to that derived by Evans and Schirmacher ${ }^{(2)}$, although there are some discrepancies in the selection of the pair potential $\phi(r)$ and the boundaries of the integral. However, we have improved their results for liquid Ar and Kr. As shown in Table 1, the calculated values of isothermal compressibilities of liquid $\mathrm{Ar}$ and $\mathrm{Kr}$ are in good agreement with the observed data. On going from liquid $\mathrm{Ne}$ to $\mathrm{Xe}$, the contribution of the first term in the denominator of eq. (29) becomes larger than the second and the third ones.

The calculated value of liquid $\mathrm{Ne}$ is about two times as large as the experimental one. For lighter atoms like $\mathrm{Ne}$, the softness of repulsive potential may be important and, therefore, the replacement of the repulsive part by a hard core will be invalid. In other words, the hard 
sphere model seems much better for the Lennard-Jones fluids of heavier atoms.

In our estimation of $\chi_{\mathrm{T}}$ of liquid metals, we have used the Hartree dielectric screening function. If another type of dielectric function is used, then the term of $1 / K_{\mathrm{TF}}$ and the last term of eq. (48) are changed, although its contribution to the compressibility is minor in comparison with other terms in the case of liquid Na-K alloys.

It may be instructive to compare the present results for the isothermal compressibility of pure liquid metals with those obtained by Bohm and Staver ${ }^{(22)}$. The sound velocity $v_{\mathrm{s}}$ in metals is given by the following equation;

$$
v_{\mathrm{s}}=(z m / 3 M)^{1 / 2} v_{\mathrm{F}},
$$

where $M, z$ and $v_{\mathrm{F}}$ are the atomic mass, valence and Fermi velocity, respectively. Using the thermodynamic relation, $\chi_{\mathrm{s}}=1 /\left(\rho v_{\mathrm{s}}^{2}\right)(\rho=M / \Omega, \Omega$; atomic volume), we have

$$
\chi_{\mathrm{s}}=\frac{3}{2 n_{\mathrm{e}} E_{\mathrm{F}}}=\frac{\left(K_{\mathrm{TF}}\right)^{2}}{4 \pi e^{2} n_{\mathrm{e}}^{2}} .
$$

This expression corresponds to the inverse of the third term in eq. (48). As seen in Table 2, the first two terms, which are related to the atomic core size, and the forth term of integral part in eq. (48), tend to be cancelled, and only the third one, which corresponds to the compressibility brought about by the existence of conduction electrons, has a main effect on the compressibility, apparently. Therefore, the calculated values of compressibility of liquid metals are almost equal to those given by only the term derived by Bohm and Staver ${ }^{(22)}$ within the present calculation.

\section{Conclusion}

In this paper we present a general formulation of isothermal compressibility in liquid binary mixture and have shown the validity of this formalism through an offering the calculated results of the Lennard-Jones fluids, those mixtures, simple liquid metals and their alloys.

In the case of Lennard-Jones fluids and those mixtures, the isothermal compressibility is expressed in terms of the short-range repulsive potential part which is replaced with the hard sphere one and the long-range attractive one which is given by the meanfield approximation.

In the expression for the isothermal compressibility of liquid metals and alloys, it is noticeable that the long wavelength limit of the pair potential can be divided into two terms as shown in eq. (47): One is ascribed to the atomic core size which is equal to the empty core radius in the present calculation, and the other is ascribed to the screening effect of conduction electrons.

\section{Acknowledgments}

The authors are grateful to Professors Y. Waseda and M. Tanaka, Tohoku University, for their kind and fruitful discussions on this work. The authors express their thanks to the Ministry of Education, Science and Culture of Japan for financial support of Grant-in-Aid.

\section{REFERENCES}

(1) N. W. Ashcroft and J. Lekner: Phys. Rev., 145 (1966), 83.

(2) R. Evans and W. Schirmacher: J. Phys. C, 11 (1978), 2437.

(3) J. D. Weeks, D. Chandler and H. C. Anderson: J. Chem. Phys., 54 (1971), 5237.

(4) M. Hasegawa and M. Watabe: J. Phys. Soc. Jpn., 32 (1972), 14.

(5) D. L. Price: Phys. Rev., A4 (1971), 358.

(6) H. Jones: Phys. Rev., A8 (1973), 3215.

(7) N. H. March and M. P. Tosi: Atomic Dynamics in Liquids, (McMillan Press, Surrey, 1976).

(8) K. Hoshino: J. Phys. F, 13 (1983), 1981.

(9) N. W. Ashcroft and D. C. Langreth: Phys. Rev., 156 (1967), 500.

(10) J. G. Kirkwood and F. P. Buff: J. Chem. Phys., 19 (1951), 774.

(11) J. P. Hansen and I. R. McDonald: Theory of Simple Liquids, (Academic Press, London, England 1976).

(12) W. C. Kerr and K. S. Singwi: Phys. Rev., A7 (1973), 1043.

(13) J. L. Yarnell, M. J. Katz, R. G. Wenzel and S. H. Koenig: Phys. Rev., A7 (1973), 2130.

(14) G. T. Clayton and L. Heaton: Phys. Rev., 121 (1961), 649.

(15) R. W. Harris and G. T. Clayton: Phys. Rev., 153 (1967), 229.

(16) N. S. Snider and T. M. Herrington: J. Chem. Phys., 47 (1967), 2248.

(17) S. P. McAlister and R. Turner: J. Phys. F: Mat. Phys., 2 (1972), L51.

(18) N. W. Ashcroft and D. Stroud: Solid State Phys., 33 
(1978), 1.

(19) I. H. Umar, I. Yokoyama and W. H. Young. Phil. Mag., 34 (1976), 535.

(20) Y Waseda: The Structure of Non-Crystalline
Materials, McGraw-Hill, New York, (1980).

(21) G. Abowitz and R. B. Gordon: J Chem. Phys., 37 (1962), 125.

(22) D. Bohm and T. Staver. Phys. Rev., 84 (1952), 836. 